\title{
$q$-Extensions for the Apostol Type Polynomials
}

\author{
Nazim I. Mahmudov and Marzieh Eini Keleshteri \\ Mathematics Department, Eastern Mediterranean University, Famagusta, Northern Cyprus, Mersin 10, Turkey \\ Correspondence should be addressed to Nazim I. Mahmudov; nazim.mahmudov@emu.edu.tr
}

Received 12 March 2014; Accepted 20 May 2014; Published 2 June 2014

Academic Editor: Necdet Batir

Copyright (c) 2014 N. I. Mahmudov and M. Eini Keleshteri. This is an open access article distributed under the Creative Commons Attribution License, which permits unrestricted use, distribution, and reproduction in any medium, provided the original work is properly cited.

The aim of this work is to introduce an extension for $q$-standard notations. The $q$-Apostol type polynomials and study some of their properties. Besides, some relations between the mentioned polynomials and some other known polynomials are obtained.

\section{Introduction, Preliminaries, and Definitions}

Throughout this research we always apply the following notations. $\mathbb{N}$ indicates the set of natural numbers, $\mathbb{N}_{0}$ indicates the set of nonnegative integers, $\mathbb{R}$ indicates the set of all real numbers, and $\mathbb{C}$ denotes the set of complex numbers. We refer the readers to [1] for all the following $q$-standard notations. The $q$-shifted factorial is defined as

$$
\begin{gathered}
(a ; q)_{0}=1, \\
(a ; q)_{n}=\prod_{j=0}^{n-1}\left(1-q^{j} a\right), \quad n \in \mathbb{N}, \\
(a ; q)_{\infty}=\prod_{j=0}^{\infty}\left(1-q^{j} a\right), \\
|q|<1, \quad a \in \mathbb{C} .
\end{gathered}
$$

The $q$-numbers and $q$-factorials are defined by

$$
\begin{array}{r}
{[a]_{q}=\frac{1-q^{a}}{1-q} \quad(q \neq 1) ;} \\
{[0] !=1 ;} \\
{[n]_{q} !=[1]_{q}[2]_{q} \cdots[n]_{q},} \\
n \in \mathbb{N}, \quad a \in \mathbb{C},
\end{array}
$$

respectively. The $q$-polynomial coefficient is defined by

$$
\left[\begin{array}{l}
n \\
k
\end{array}\right]_{q}=\frac{[n]_{q} !}{[k]_{q} ![n-k]_{q} !} .
$$

The $q$-analogue of the function $(x+y)^{n}$ is defined by

$$
(x+y)_{q}^{n}:=\sum_{k=0}^{n}\left[\begin{array}{l}
n \\
k
\end{array}\right]_{q} q^{(1 / 2) k(k-1)} x^{n-k} y^{k}, \quad n \in \mathbb{N}_{0} .
$$

The $q$-binomial formula is known as

$$
(1-a)_{q}^{n}=\prod_{j=0}^{n-1}\left(1-q^{j} a\right)=\sum_{k=0}^{n}\left[\begin{array}{l}
n \\
k
\end{array}\right]_{q} q^{(1 / 2) k(k-1)}(-1)^{k} a^{k} .
$$

In the standard approach to the $q$-calculus, two exponential functions are used:

$$
\begin{aligned}
& e_{q}(z)=\sum_{n=0}^{\infty} \frac{z^{n}}{[n]_{q} !}=\prod_{j=0}^{\infty} \frac{1}{\left(1-(1-q) q^{j} z\right)}, \\
& \quad 0<|q|<1, \quad|z|<\frac{1}{|1-q|}, \\
& E_{q}(z)=\sum_{k=0}^{\infty} \frac{q^{(1 / 2) k(k-1)} z^{k}}{[k]_{q} !}=\prod_{j=0}^{\infty}\left(1+(1-q) q^{j} z\right), \\
& \quad 0<|q|<1, \quad z \in \mathbb{C} .
\end{aligned}
$$


As an immediate result of these two definitions, we have $e_{q}(z) E_{q}(-z)=1$.

Recently, Luo and Srivastava [2] introduced and studied the generalized Apostol-Bernoulli polynomials $B_{n}^{\alpha}(x ; \lambda)$ and the generalized Apostol-Euler polynomials $E_{n}^{\alpha}(x ; \lambda)$. Kurt [3] gave the generalization of the Bernoulli polynomials $B_{n}^{[m-1, \alpha]}(x)$ of order $\alpha$ and studied their properties. They also studied these polynomials systematically; see [2, 4-9]. There are numerous recent investigations on this subject by many other authors; see [3, 10-20]. More recently, Tremblay et al. [10] further gave the definition of $B_{n}^{[m-1, \alpha]}(x ; \lambda)$ and studied their properties. On the other hand, Mahmudov and Keleshteri $[21,22]$ studied various two dimensional $q$-polynomials. Motivated by these papers, we define generalized Apostol type q-polynomials as follows.

Definition 1. Let $q, \alpha \in \mathbb{C}, m \in \mathbb{N}$, and $0<|q|<1$. The generalized $q$-Apostol-Bernoulli numbers $B_{n, q}^{[m-1, \alpha]}$ and polynomials $B_{n, q}^{[m-1, \alpha]}(x, y ; \lambda)$ in $x, y$ of order $\alpha$ are defined, in a suitable neighborhood of $t=0$, by means of the generating functions:

$$
\begin{gathered}
\left(\frac{t^{m}}{\lambda e_{q}(t)-T_{m-1, q}(t)}\right)^{\alpha}=\sum_{n=0}^{\infty} B_{n, q}^{[m-1, \alpha]}(\lambda) \frac{t^{n}}{[n]_{q} !}, \\
\left(\frac{t^{m}}{\lambda e_{q}(t)-T_{m-1, q}(t)}\right)^{\alpha} e_{q}(t x) E_{q}(t y) \\
=\sum_{n=0}^{\infty} B_{n, q}^{[m-1, \alpha]}(x, y ; \lambda) \frac{t^{n}}{[n]_{q} !}
\end{gathered}
$$

where $T_{m-1, q}(t)=\sum_{k=0}^{m-1}\left(t^{k} /[k] q !\right)$.

Definition 2. Let $q, \alpha \in \mathbb{C}, 0<|q|<1$, and $m \in$ $\mathbb{N}$. The generalized $q$-Apostol-Euler numbers $E_{n, q}^{[m-1, \alpha]}$ and polynomials $E_{n, q}^{[m-1, \alpha]}(x, y ; \lambda)$ in $x, y$ of order $\alpha$ are defined, in a suitable neighborhood of $t=0$, by means of the generating functions:

$$
\begin{gathered}
\left(\frac{2^{m}}{\lambda e_{q}(t)+T_{m-1, q}(t)}\right)^{\alpha}=\sum_{n=0}^{\infty} E_{n, q}^{[m-1, \alpha]}(\lambda) \frac{t^{n}}{[n]_{q} !}, \\
\left(\frac{2^{m}}{\lambda e_{q}(t)+T_{m-1, q}(t)}\right)^{\alpha} e_{q}(t x) E_{q}(t y) \\
=\sum_{n=0}^{\infty} E_{n, q}^{[m-1, \alpha]}(x, y ; \lambda) \frac{t^{n}}{[n]_{q} !} .
\end{gathered}
$$

Definition 3. Let $q, \alpha \in \mathbb{C}, 0<|q|<1$, and $m \in \mathbb{N}$. The generalized $q$-Apostol-Genocchi numbers $G_{n, q}^{[m-1, \alpha]}$ and polynomials $G_{n, q}^{[m-1, \alpha]}(x, y ; \lambda)$ in $x, y$ of order $\alpha$ are defined, in a suitable neighborhood of $t=0$, by means of the generating functions:

$$
\begin{gathered}
\left(\frac{2^{m} t^{m}}{\lambda e_{q}(t)+T_{m-1, q}(t)}\right)^{\alpha}=\sum_{n=0}^{\infty} G_{n, q}^{[m-1, \alpha]}(\lambda) \frac{t^{n}}{[n]_{q} !} \\
\left(\frac{2^{m} t^{m}}{\lambda e_{q}(t)+T_{m-1, q}(t)}\right)^{\alpha} e_{q}(t x) E_{q}(t y) \\
=\sum_{n=0}^{\infty} G_{n, q}^{[m-1, \alpha]}(x, y ; \lambda) \frac{t^{n}}{[n]_{q} !}
\end{gathered}
$$

Clearly, for $m=1$, one has

$$
\begin{aligned}
& B_{n, q}^{[0, \alpha]}(x, y ; \lambda)=B_{n, q}^{(\alpha)}(x, y ; \lambda), \\
& E_{n, q}^{[0, \alpha]}(x, y ; \lambda)=E_{n, q}^{(\alpha)}(x, y ; \lambda), \\
& G_{n, q}^{[0, \alpha]}(x, y ; \lambda)=G_{n, q}^{(\alpha)}(x, y ; \lambda) .
\end{aligned}
$$

For $m=1$ and $\lambda=1$, one has

$$
\begin{aligned}
& B_{n, q}^{[0, \alpha]}(x, y ; 1)=B_{n, q}^{(\alpha)}(x, y), \\
& E_{n, q}^{[0, \alpha]}(x, y ; 1)=E_{n, q}^{(\alpha)}(x, y), \\
& G_{n, q}^{[0, \alpha]}(x, y ; 1)=G_{n, q}^{(\alpha)}(x, y) .
\end{aligned}
$$

For $x=y=0$, one has

$$
\begin{aligned}
& B_{n, q}^{[m-1, \alpha]}(0,0 ; \lambda)=B_{n, q}^{[m-1, \alpha]}(\lambda), \\
& E_{n, q}^{[m-1, \alpha]}(0,0 ; \lambda)=E_{n, q}^{[m-1, \alpha]}(\lambda), \\
& G_{n, q}^{[m-1, \alpha]}(0,0 ; \lambda)=G_{n, q}^{[m-1, \alpha]}(\lambda) .
\end{aligned}
$$

\section{Properties of the Apostol Type $q$-Polynomials}

In this section, we show some basic properties of the generalized q-polynomials. We only prove the facts for one of them. Obviously, by applying the similar technique, other ones can be proved. 
Proposition 4. The generalized q-polynomials $B_{n, q}^{[m-1, \alpha]}(x$, $y ; \lambda), E_{n, q}^{[m-1, \alpha]}(x, y ; \lambda)$, and $G_{n, q}^{[m-1, \alpha]}(x, y ; \lambda)$ satisfy the following relations:

$$
\begin{aligned}
& B_{n, q}^{[m-1, \alpha+\beta]}(x, y ; \lambda) \\
& =\sum_{k=0}^{n}\left[\begin{array}{l}
n \\
k
\end{array}\right]_{q} B_{k, q}^{[m-1, \alpha]}(x, 0 ; \lambda) \\
& \quad \times B_{n-k, q}^{[m-1, \beta]}(0, y ; \lambda), \\
& E_{n, q}^{[m-1, \alpha+\beta]}(x, y ; \lambda) \\
& =\sum_{k=0}^{n}\left[\begin{array}{l}
n \\
k
\end{array}\right]_{q} E_{k, q}^{[m-1, \alpha]}(x, 0 ; \lambda) \\
& \quad \times E_{n-k, q}^{[m-1, \beta]}(0, y ; \lambda), \\
& G_{n, q}^{[m-1, \alpha+\beta]}(x, y ; \lambda) \\
& =\sum_{k=0}^{n}\left[\begin{array}{l}
n \\
k
\end{array}\right]_{q} G_{k, q}^{[m-1, \alpha]}(x, 0 ; \lambda) \\
& \quad \times G_{n-k, q}^{[m-1, \beta]}(0, y ; \lambda) .
\end{aligned}
$$

Proof. We only prove the second identity. By using Definition 2, we have

$$
\begin{aligned}
\sum_{n=0}^{\infty} & E_{n, q}^{[m-1, \alpha+\beta]}(x, y ; \lambda) \frac{t^{n}}{[n]_{q} !} \\
= & \left(\frac{2^{m}}{\lambda e_{q}(t)+T_{m-1, q}(t)}\right)^{\alpha+\beta} e_{q}(t x) E_{q}(t y) \\
= & \left(\frac{2^{m}}{\lambda e_{q}(t)+T_{m-1, q}(t)}\right)^{\alpha} e_{q}(t x) \\
& \times\left(\frac{2^{m}}{\lambda e_{q}(t)+T_{m-1, q}(t)}\right)^{\beta} E_{q}(t y) \\
= & \sum_{n=0}^{\infty} E_{n, q}^{[m-1, \alpha]}(x, 0 ; \lambda) \frac{t^{n}}{[n]_{q} !} \\
& \times \sum_{n=0}^{\infty} E_{n, q}^{[m-1, \beta]}(0, y ; \lambda) \frac{t^{n}}{[n]_{q} !} \\
= & \sum_{n=0}^{\infty} \sum_{k=0}^{n}\left[\begin{array}{l}
n \\
k
\end{array}\right] E_{q}^{[m-1, \alpha]}(x, 0 ; \lambda) E_{n-k, q}^{[m-1, \beta]}(0, y ; \lambda) \frac{t^{n}}{[n]_{q} !} .
\end{aligned}
$$

Comparing the coefficients of the term $t^{n} /[n]_{q}$ ! in both sides gives the result.
Corollary 5. The generalized q-polynomials $B_{n, q}^{[m-1, \alpha]}(x, y ; \lambda)$, $E_{n, q}^{[m-1, \alpha]}(x, y ; \lambda)$, and $G_{n, q}^{[m-1, \alpha]}(x, y ; \lambda)$ satisfy the following relations:

$$
\begin{aligned}
B_{n, q}^{[m-1, \alpha]}(x, y ; \lambda) & =\sum_{k=0}^{n}\left[\begin{array}{l}
n \\
k
\end{array}\right]_{q} B_{k, q}^{[m-1, \alpha]}(0, y ; \lambda) x^{n-k}, \\
E_{n, q}^{[m-1, \alpha]}(x, y ; \lambda) & =\sum_{k=0}^{n}\left[\begin{array}{l}
n \\
k
\end{array}\right]_{q} E_{k, q}^{[m-1, \alpha]}(0, y ; \lambda) x^{n-k}, \\
G_{n, q}^{[m-1, \alpha]}(x, y ; \lambda) & =\sum_{k=0}^{n}\left[\begin{array}{l}
n \\
k
\end{array}\right]_{q} G_{k, q}^{[m-1, \alpha]}(0, y ; \lambda) x^{n-k} .
\end{aligned}
$$

Proposition 6. The generalized q-polynomials $B_{n, q}^{[m-1, \alpha]}(x, y$; $\lambda), E_{n, q}^{[m-1, \alpha]}(x, y ; \lambda)$, and $G_{n, q}^{[m-1, \alpha]}(x, y ; \lambda)$ satisfy the following relations:

$$
\begin{aligned}
& \lambda B_{n, q}^{[m-1, \alpha]}(1, y ; \lambda)-B_{n, q}^{[m-1, \alpha]}(0, y ; \lambda) \\
& =\sum_{k=0}^{n}\left[\begin{array}{l}
n \\
k
\end{array}\right]_{q}[k]_{q} B_{k, q}^{[m-1, \alpha]}(0, y ; \lambda) B_{n-k, q}^{[0,-1]}(\lambda), \quad \text { for } n \geq 1, \\
& \lambda E_{n, q}^{[m-1, \alpha]}(1, y ; \lambda)+E_{n, q}^{[m-1, \alpha]}(0, y ; \lambda) \\
& \quad=2 \sum_{k=0}^{n}\left[\begin{array}{l}
n \\
k
\end{array}\right]_{q}^{[m-1, \alpha]}(0, y ; \lambda) E_{n-k, q}^{[0,-1]}(\lambda), \\
& \lambda G_{n, q}^{[m-1, \alpha]}(1, y ; \lambda)+G_{n, q}^{[m-1, \alpha]}(0, y ; \lambda) \\
& \quad=2 \sum_{k=0}^{n}\left[\begin{array}{l}
n \\
k
\end{array}\right]_{q}[k]_{q} G_{k, q}^{[m-1, \alpha]}(0, y ; \lambda) G_{n-k, q}^{[0,-1]}(\lambda), \quad \text { for } n \geq 1 .
\end{aligned}
$$

Proof. We only prove (18). By using Definition 2 and starting from the left hand side of the relation (18), we have

$$
\begin{aligned}
\sum_{n=0}^{\infty}( & \left.\lambda E_{n, q}^{[m-1, \alpha]}(1, y ; \lambda)+E_{n, q}^{[m-1, \alpha]}(0, y ; \lambda)\right) \frac{t^{n}}{[n]_{q} !} \\
= & \lambda\left(\frac{2^{m}}{\lambda e_{q}(t)+T_{m-1, q}(t)}\right)^{\alpha} e_{q}(t) E_{q}(t y) \\
& +\left(\frac{2^{m}}{\lambda e_{q}(t)+T_{m-1, q}(t)}\right)^{\alpha} E_{q}(t y) \\
= & \left(\frac{2^{m}}{\lambda e_{q}(t)+T_{m-1, q}(t)}\right)^{\alpha} E_{q}(t y)\left(\lambda e_{q}(t)+1\right) \\
= & 2\left(\frac{2^{m}}{\lambda e_{q}(t)+T_{m-1, q}(t)}\right)^{\alpha} E_{q}(t y)\left(\frac{2}{\lambda e_{q}(t)+1}\right)^{-1} \\
= & 2 \sum_{n=0}^{\infty} E_{n, q}^{[m-1, \alpha]}(0, y ; \lambda) \frac{t^{n}}{[n]_{q} !} \sum_{n=0}^{\infty} E_{n, q}^{[0,-1]}(\lambda) \frac{t^{n}}{[n]_{q} !}
\end{aligned}
$$




$$
=2 \sum_{n=0}^{\infty} \sum_{k=0}^{n}\left[\begin{array}{l}
n \\
k
\end{array}\right]_{q} E_{k, q}^{[m-1, \alpha]}(0, y ; \lambda) E_{n-k, q}^{[0,-1]}(\lambda) \frac{t^{n}}{[n]_{q} !} .
$$

Comparing the coefficients of the term $t^{n} /[n]_{q}$ ! in both sides gives the result.

\section{3. q-Analogue of the Luo-Srivastava Addition Theorem}

In this section, we state and prove a $q$-generalization of the Luo-Srivastava addition theorem.

Theorem 7. The following relation holds between generalized q-Apostol-Euler and q-Apostol-Bernoulli polynomials:

$$
\begin{aligned}
& E_{n, q}^{[m-1, \alpha]}(x, y ; \lambda) \\
& =\sum_{j=0}^{n} \frac{1}{[n+1]_{q}}\left[\begin{array}{c}
n+1 \\
j
\end{array}\right]_{q} \\
& \quad \times\left(\lambda \sum_{k=0}^{n-j+1} \frac{1}{[n+1]_{q}}\left[\begin{array}{c}
n-j+1 \\
k
\end{array}\right]_{q} E_{k, q}^{[m-1, \alpha-1]}(0, y ; \lambda)\right. \\
& \left.\quad-E_{n-j+1, q}^{[m-1, \alpha]}(0, y ; \lambda)\right) \\
& \quad \times B_{j, q}(x, 0 ; \lambda)+\frac{\lambda-1}{[n+1]_{q}} \\
& \quad \times\left(\frac{2^{m}}{\lambda+1}\right)^{\alpha} B_{n+1, q}(x, 0 ; \lambda) .
\end{aligned}
$$

Proof. We take aid of the following identity to prove (21):

$$
\begin{gathered}
\lambda \frac{t}{\lambda e_{q}(t)-1} e_{q}(t x) e_{q}(t)-\frac{t}{\lambda e_{q}(t)-1} e_{q}(t x) \\
=\frac{t e_{q}(t x)}{\lambda e_{q}(t)-1}\left(\lambda e_{q}(t)-1\right)=t e_{q}(t x) .
\end{gathered}
$$

Therefore, we can write

$$
\begin{aligned}
\lambda & \sum_{n=0}^{\infty} \sum_{k=0}^{n}\left[\begin{array}{l}
n \\
k
\end{array}\right]_{q} B_{k, q}(x, 0 ; \lambda) \frac{t^{n}}{[n]_{q} !}-\sum_{n=0}^{\infty} B_{n, q}(x, 0 ; \lambda) \frac{t^{n}}{[n]_{q} !} \\
& =\sum_{n=0}^{\infty} x^{n} \frac{t^{n+1}}{[n+1]_{q} !}[n+1]_{q} \\
& =\sum_{n=0}^{\infty}[n]_{q} x^{n-1} \frac{t^{n}}{[n]_{q} !} .
\end{aligned}
$$

From that we can conclude the following:

$$
\lambda \sum_{k=0}^{n}\left[\begin{array}{l}
n \\
k
\end{array}\right]_{q} B_{k, q}(x, 0 ; \lambda)-B_{n, q}(x, 0 ; \lambda)=[n]_{q} x^{n-1} .
$$

That is,

$$
x^{n}=\frac{1}{[n+1]_{q}}\left(\lambda \sum_{k=0}^{n+1}\left[\begin{array}{c}
n+1 \\
k
\end{array}\right]_{q} B_{k, q}(x, 0 ; \lambda)-B_{n+1, q}(x, 0 ; \lambda)\right) .
$$

Substituting (25) into the right hand side of (16), we obtain

$$
\begin{aligned}
& E_{n, q}^{[m-1, \alpha]}(x, y ; \lambda) \\
& =\sum_{k=0}^{n}\left[\begin{array}{l}
n \\
k
\end{array}\right]_{q} E_{k, q}^{[m-1, \alpha]}(0, y ; \lambda) \frac{1}{[n-k+1]_{q}} \\
& \times\left(\lambda \sum_{j=0}^{n-k+1}\left[\begin{array}{c}
n-k+1 \\
j
\end{array}\right]_{q} B_{j, q}(x, 0 ; \lambda)\right. \\
& \left.-B_{n-k+1, q}(x, 0 ; \lambda)\right) \\
& =\sum_{k=0}^{n}\left[\begin{array}{l}
n \\
k
\end{array}\right]_{q} E_{k, q}^{[m-1, \alpha]}(0, y ; \lambda) \frac{1}{[n-k+1]_{q}} \\
& \times\left(\lambda \sum_{j=0}^{n-k}\left[\begin{array}{c}
n-k+1 \\
j
\end{array}\right]_{q} B_{j, q}(x, 0 ; \lambda)\right. \\
& \left.+(\lambda-1) B_{n-k+1, q}(x, 0 ; \lambda)\right) \\
& =\sum_{k=0}^{n}\left[\begin{array}{l}
n \\
k
\end{array}\right]_{q} E_{k, q}^{[m-1, \alpha]}(0, y ; \lambda) \frac{\lambda}{[n-k+1]_{q}} \\
& \times \sum_{j=0}^{n-k}\left[\begin{array}{c}
n-k+1 \\
j
\end{array}\right]_{q} B_{j, q}(x, 0 ; \lambda) \\
& +\sum_{k=0}^{n}\left[\begin{array}{l}
n \\
k
\end{array}\right]_{q} E_{k, q}^{[m-1, \alpha]}(0, y ; \lambda) \frac{\lambda-1}{[n-k+1]_{q}} \\
& \times B_{n-k+1, q}(x, 0 ; \lambda):=I_{1}+I_{2} .
\end{aligned}
$$

Thus, from one hand, we can write

$$
\begin{aligned}
I_{1}= & \sum_{k=0}^{n}\left[\begin{array}{l}
n \\
k
\end{array}\right]_{q} E_{k, q}^{[m-1, \alpha]}(0, y ; \lambda) \frac{\lambda}{[n-k+1]_{q}} \\
& \times \sum_{j=0}^{n-k}\left[\begin{array}{c}
n-k+1 \\
j
\end{array}\right]_{q} B_{j, q}(x, 0 ; \lambda) \\
= & \sum_{j=0}^{n} \sum_{k=0}^{n-j} \frac{\lambda}{[n+1]_{q}}\left[\begin{array}{c}
n+1 \\
n-k+1
\end{array}\right]_{q}\left[\begin{array}{c}
n-k+1 \\
j
\end{array}\right]_{q} \\
& \times E_{k, q}^{[m-1, \alpha]}(0, y ; \lambda) B_{j, q}(x, 0 ; \lambda) .
\end{aligned}
$$

As we know that

$$
\left[\begin{array}{c}
m \\
l
\end{array}\right]_{q}\left[\begin{array}{l}
l \\
n
\end{array}\right]_{q}=\left[\begin{array}{c}
m \\
n
\end{array}\right]_{q}\left[\begin{array}{c}
m-n \\
m-l
\end{array}\right]_{q}, \quad \text { for } m \geq l \geq n,
$$


we can continue as

$$
\begin{aligned}
I_{1}= & \sum_{j=0}^{n} \sum_{k=0}^{n-j} \frac{\lambda}{[n+1]_{q}}\left[\begin{array}{c}
n+1 \\
j
\end{array}\right]_{q}\left[\begin{array}{c}
n-j+1 \\
k
\end{array}\right]_{q} \\
& \times E_{k, q}^{[m-1, \alpha]}(0, y ; \lambda) B_{j, q}(x, 0 ; \lambda) \\
= & \sum_{j=0}^{n} \frac{\lambda}{[n+1]_{q}}\left[\begin{array}{c}
n+1 \\
j
\end{array}\right]_{q} B_{j, q}(x, 0 ; \lambda) \\
& \times \sum_{k=0}^{n-j}\left[\begin{array}{c}
n-j+1 \\
k
\end{array} E_{q}^{[m-1, \alpha]}(0, y ; \lambda)\right. \\
= & \sum_{j=0}^{n} \frac{\lambda}{[n+1]_{q}}\left[\begin{array}{c}
n+1 \\
j
\end{array}\right]_{q} B_{j, q}(x, 0 ; \lambda) \\
& \times\left(E_{n-j+1, q}^{[m-1, \alpha]}(1, y ; \lambda)-E_{n-j+1, q}^{[m-1, \alpha]}(0, y ; \lambda)\right) .
\end{aligned}
$$

On the other hand, for $I_{2}$, we can write

$$
\begin{aligned}
I_{2}= & \sum_{k=0}^{n}\left[\begin{array}{l}
n \\
k
\end{array}\right]_{q} E_{k, q}^{[m-1, \alpha]}(0, y ; \lambda) \frac{\lambda-1}{[n-k+1]_{q}} B_{n-k+1, q}(x, 0 ; \lambda) \\
= & \sum_{k=0}^{n}\left[\begin{array}{c}
n+1 \\
k
\end{array}\right]_{q} \frac{\lambda-1}{[n+1]_{q}} B_{n-k+1, q}(x, 0 ; \lambda) E_{k, q}^{[m-1, \alpha]}(0, y ; \lambda) \\
= & \sum_{k=0}^{n+1}\left[\begin{array}{c}
n+1 \\
k
\end{array}\right]_{q} \frac{\lambda-1}{[n+1]_{q}} B_{n-k+1, q}(x, 0 ; \lambda) E_{k, q}^{[m-1, \alpha]}(0, y ; \lambda) \\
& -\frac{\lambda-1}{[n+1]_{q}} B_{0, q}(x, 0 ; \lambda) E_{k, q}^{[m-1, \alpha]}(0, y ; \lambda),
\end{aligned}
$$

and, as $B_{0, q}(x, 0 ; \lambda)=0$, we have

$$
\begin{aligned}
I_{2} & =\sum_{k=0}^{n+1}\left[\begin{array}{c}
n+1 \\
k
\end{array}\right]_{q} \frac{\lambda-1}{[n+1]_{q}} B_{n-k+1, q}(x, 0 ; \lambda) E_{k, q}^{[m-1, \alpha]}(0, y ; \lambda) \\
= & \sum_{j=0}^{n+1}\left[\begin{array}{c}
n+1 \\
j
\end{array}\right]_{q} \frac{\lambda-1}{[n+1]_{q}} B_{j, q}(x, 0 ; \lambda) E_{n-j+1, q}^{[m-1, \alpha]}(0, y ; \lambda) \\
= & \sum_{j=0}^{n}\left[\begin{array}{c}
n+1 \\
j
\end{array}\right]_{q} \frac{\lambda-1}{[n+1]_{q}} B_{j, q}(x, 0 ; \lambda) E_{n-j+1, q}^{[m-1, \alpha]}(0, y ; \lambda) \\
& +\frac{\lambda-1}{[n+1]_{q}} B_{n+1, q}(x, 0 ; \lambda) E_{0, q}^{[m-1, \alpha]}(0, y ; \lambda) .
\end{aligned}
$$

Adding $I_{2}$ to $I_{1}$ we obtain

$$
\begin{aligned}
& E_{n, q}^{[m-1, \alpha]}(x, y ; \lambda) \\
& \quad=I_{1}+I_{2} \\
& \quad=\sum_{j=0}^{n} \frac{\lambda}{[n+1]_{q}}\left[\begin{array}{c}
n+1 \\
j
\end{array}\right]_{q} B_{j, q}(x, 0 ; \lambda) \\
& \quad \times\left(E_{n-j+1, q}^{[m-1, \alpha]}(1, y ; \lambda)-E_{n-j+1, q}^{[m-1, \alpha]}(0, y ; \lambda)\right) \\
& \quad+\sum_{j=0}^{n}\left[\begin{array}{c}
n+1 \\
j
\end{array}\right]_{q} \frac{\lambda-1}{[n+1]_{q}} B_{j, q}(x, 0 ; \lambda) E_{n-j+1, q}^{[m-1, \alpha]}(0, y ; \lambda) \\
& \quad+\frac{\lambda-1}{[n+1]_{q}} B_{n+1, q}(x, 0 ; \lambda) E_{0, q}^{[m-1, \alpha]}(0, y ; \lambda) .
\end{aligned}
$$

\section{Consequently,}

$$
\begin{aligned}
& E_{n, q}^{[m-1, \alpha]}(x, y ; \lambda) \\
& =\sum_{j=0}^{n} \frac{1}{[n+1]_{q}}\left[\begin{array}{c}
n+1 \\
j
\end{array}\right]_{q} \\
& \times\left(\lambda E_{n-j+1, q}^{[m-1, \alpha]}(1, y ; \lambda)-\lambda E_{n-j+1, q}^{[m-1, \alpha]}(0, y ; \lambda)\right. \\
& \left.+(\lambda-1) E_{n-j+1, q}^{[m-1, \alpha]}(0, y ; \lambda)\right) \\
& \times B_{j, q}(x, 0 ; \lambda)+\frac{\lambda-1}{[n+1]_{q}} \\
& \times B_{n+1, q}(x, 0 ; \lambda) E_{0, q}^{[m-1, \alpha]}(0, y ; \lambda) \\
& =\sum_{j=0}^{n} \frac{1}{[n+1]_{q}}\left[\begin{array}{c}
n+1 \\
j
\end{array}\right]_{q} \\
& \times\left(\lambda E_{n-j+1, q}^{[m-1, \alpha]}(1, y ; \lambda)-E_{n-j+1, q}^{[m-1, \alpha]}(0, y ; \lambda)\right) \\
& \times B_{j, q}(x, 0 ; \lambda)+\frac{(\lambda-1)}{[n+1]_{q}} B_{n+1, q}(x, 0 ; \lambda) \\
& \times E_{0, q}^{[m-1, \alpha]}(0, y ; \lambda) \\
& =\sum_{j=0}^{n} \frac{1}{[n+1]_{q}}\left[\begin{array}{c}
n+1 \\
j
\end{array}\right]_{q} \\
& \times\left(\lambda \sum_{k=0}^{n-j+1}\left[\begin{array}{c}
n-j+1 \\
k
\end{array}\right]_{q} E_{n-j+1, q}^{[m-1, \alpha]}(0, y ; \lambda)\right. \\
& \left.-E_{n-j+1, q}^{[m-1, \alpha]}(0, y ; \lambda)\right) \\
& \times B_{j, q}(x, 0 ; \lambda)+\frac{(\lambda-1)}{[n+1]_{q}}\left(\frac{2^{m}}{\lambda+1}\right)^{\alpha} B_{n+1, q}(x, 0 ; \lambda) .
\end{aligned}
$$


Taking $m=1$ in Theorem 7, we get a $q$-generalization of the Luo-Srivastava addition theorem [2].

Corollary 8. The following relation holds between generalized q-Apostol-Euler and q-Apostol-Bernoulli polynomials:

$$
\begin{aligned}
E_{n, q}^{(\alpha)}(x, y ; \lambda)= & \sum_{j=0}^{n} \frac{2}{[j+1]_{q}}\left[\begin{array}{c}
n \\
j
\end{array}\right]_{q} \\
& \times\left(E_{j+1, q}^{(\alpha)}(0, y ; \lambda)-E_{j+1, q}^{(\alpha)}(0, y ; \lambda)\right) \\
& \times B_{n-j, q}(x, 0 ; \lambda)+\frac{\lambda-1}{[n+1]_{q}}\left(\frac{2}{\lambda+1}\right)^{\alpha} \\
& \times B_{n+1, q}(x, 0 ; \lambda) .
\end{aligned}
$$

Letting $q \uparrow 1$, we get the Luo-Srivastava addition theorem (see $[12])$ :

$$
\begin{aligned}
E_{n}^{(\alpha)}(x+y ; \lambda)= & \sum_{j=0}^{n} \frac{2}{j+1}\left(\begin{array}{c}
n \\
j
\end{array}\right) \\
& \times\left(E_{j+1}^{(\alpha)}(y ; \lambda)-E_{j+1}^{(\alpha)}(y ; \lambda)\right) \\
& \times B_{n-j, q}(x ; \lambda)+\frac{\lambda-1}{n+1}\left(\frac{2}{\lambda+1}\right)^{\alpha} \\
& \times B_{n+1}(x ; \lambda) .
\end{aligned}
$$

Next theorem gives relationship between $E_{n, q}^{[m-1, \alpha]}(x, y ; \lambda)$ and $G_{n, q}(x, 0)$.

Theorem 9. The following relation holds between generalized q-Apostol-Euler and q-Apostol-Genocchi polynomials:

$$
\begin{aligned}
E_{n, q}^{[m-1, \alpha]}(x, y ; \lambda)= & \frac{1}{2} \sum_{k=0}^{n} \frac{1}{[k+1]_{q}} \\
& \times\left(\lambda \sum_{j=k}^{n}\left[\begin{array}{l}
n \\
j
\end{array}\right]_{q}\left[\begin{array}{l}
j \\
k
\end{array}\right]_{q} E_{n-j, q}^{[m-1, \alpha]}(0, y ; \lambda)\right. \\
& \left.\quad+\sum_{k=0}^{n}\left[\begin{array}{l}
n \\
k
\end{array}\right]_{q} E_{n-k, q}^{[m-1, \alpha]}(0, y ; \lambda)\right) \\
& \times G_{k+1, q}(x, 0) .
\end{aligned}
$$

Proof. The proof follows from the following identity:

$$
\begin{aligned}
& \left(\frac{2^{m}}{\lambda e_{q}(t)+T_{m-1, q}(t)}\right)^{\alpha} e_{q}(t x) E_{q}(t y) \\
& =\left(\frac{2^{m}}{\lambda e_{q}(t)+T_{m-1, q}(t)}\right)^{\alpha} E_{q}(t y) \frac{2 t}{e_{q}(t)+1} \\
& \quad \times e_{q}(t x) \frac{e_{q}(t)+1}{2 t} .
\end{aligned}
$$

Theorem 10. The following relation holds between generalized $q$-Apostol-Euler and q-Stirling polynomials $S_{q}(i, j)$ of the second kind:

$$
\begin{aligned}
E_{n, q}^{[m-1, \alpha]}(x, y ; \lambda)= & \sum_{k=0}^{n} \sum_{j=k}^{n}\left[\begin{array}{c}
n \\
n-j
\end{array}\right]_{q} \\
& \times E_{n-j, q}^{[m-1, \alpha]}(0, y ; \lambda) S_{q}(j, k) x_{k}(x) .
\end{aligned}
$$

Proof. The $q$-Stirling polynomials $S_{q}(n, k)$ of the second kind are defined by means of the following generating function:

$$
x^{n}=\sum_{k=0}^{n} S_{q}(n, k) x_{k}(x),
$$

where $x_{k}(x)=x\left(x-[1]_{q}\right)\left(x-[2]_{q}\right) \cdots\left(x-[k-1]_{q}\right)$; see [23]. Replacing identity (39) in the right hand side of (16), we have

$$
\begin{aligned}
E_{n, q}^{[m-1, \alpha]}(x, y ; \lambda)= & \sum_{k=0}^{n}\left[\begin{array}{l}
n \\
k
\end{array}\right]_{q} E_{k, q}^{[m-1, \alpha]}(0, y ; \lambda) \\
& \times \sum_{k=0}^{n-k} S_{q}(n-k, k) x_{k}(x) \\
= & \sum_{k=0}^{n} \sum_{j=k}^{n}\left[\begin{array}{c}
n \\
n-j
\end{array}\right]_{q} \\
& \times E_{n-j, q}^{[m-1, \alpha]}(0, y ; \lambda) S_{q}(j, k) x_{k}(x) .
\end{aligned}
$$

Theorem 11. The relationship

$$
\begin{aligned}
E_{n, q}^{[m-1, \alpha]}(x, y ; \lambda)= & \sum_{k=0}^{[n / 2]} \sum_{j=0}^{n-2 k}\left[\begin{array}{l}
n \\
k
\end{array}\right]_{q}\left[\begin{array}{c}
n-2 k \\
j
\end{array}\right]_{q} \frac{[k]_{q} !}{[2]_{q}^{n}[k]_{q} !} \\
& \times E_{j, q}^{[m-1, \alpha]}(0, y ; \lambda) H_{n-2 k-j, q}(x)
\end{aligned}
$$

holds between the polynomials $E_{n, q}^{[m-1, \alpha]}(x, y ; \lambda)$ and the $q$ Hermite polynomials defined by (see [24])

$$
e_{q}(t x) E_{q^{2}}\left(-\frac{t^{2}}{[2]_{q}}\right)=\sum_{n=0}^{\infty} H_{n, q}(x) \frac{t^{n}}{[n]_{q} !} .
$$


Proof. Indeed,

$$
\begin{aligned}
& \sum_{n=0}^{\infty} E_{n, q}^{[m-1, \alpha]}(x, y ; \lambda) \frac{t^{n}}{[n]_{q} !} \\
& =\left(\frac{2^{m}}{\lambda e_{q}(t)+T_{m-1, q}(t)}\right)^{\alpha} e_{q}(t x) E_{q}(t y) \\
& =\left(\frac{2^{m}}{\lambda e_{q}(t)+T_{m-1, q}(t)}\right)^{\alpha} E_{q}(t y) e_{q}(t x) \\
& \times E_{q^{2}}\left(-\frac{t^{2}}{[2]_{q}}\right) e_{q^{2}}\left(\frac{t^{2}}{[2]_{q}}\right) \\
& =\sum_{n=0}^{\infty} E_{n, q}^{[m-1, \alpha]}(0, y ; \lambda) \frac{t^{n}}{[n]_{q} !} \\
& \times \sum_{n=0}^{\infty} H_{n, q}(x) \frac{t^{n}}{[n]_{q} !} \sum_{n=0}^{\infty} \frac{t^{2 n}}{[2]_{q}^{n}[n]_{q^{2}} !} \\
& =\sum_{n=0}^{\infty} \sum_{j=0}^{n}\left[\begin{array}{c}
n \\
j
\end{array}\right]_{q} E_{j, q}^{[m-1, \alpha]}(0, y ; \lambda) H_{n-j, q}(x) \frac{t^{n}}{[n]_{q} !} \\
& \times \sum_{n=0}^{\infty} \frac{t^{2 n}}{[2]_{q}^{n}[n]_{q^{2}} !} \\
& =\sum_{n=0}^{\infty} \sum_{k=0}^{[n / 2]} \frac{[n]_{q} !}{[2]_{q}^{n}[k]_{q^{2}} ![n-2 k]_{q} !} \\
& \times \sum_{j=0}^{n-2 k}\left[\begin{array}{c}
n-2 k \\
j
\end{array}\right]_{q} E_{j, q}^{[m-1, \alpha]}(0, y ; \lambda) H_{n-2 k-j, q}(x) \frac{t^{n}}{[n]_{q} !} \\
& =\sum_{n=0}^{\infty} \sum_{k=0}^{[n / 2]} \sum_{j=0}^{n-2 k}\left[\begin{array}{l}
n \\
k
\end{array}\right]_{q}\left[\begin{array}{c}
n-2 k \\
j
\end{array}\right]_{q} \frac{[k]_{q} !}{[2]_{q}^{n}[k]_{q^{2}} !} \\
& \times E_{j, q}^{[m-1, \alpha]}(0, y ; \lambda) H_{n-2 k-j, q}(x) \frac{t^{n}}{[n]_{q} !} .
\end{aligned}
$$

\section{Conflict of Interests}

The authors declare that there is no conflict of interests regarding the publication of this paper.

\section{References}

[1] G. E. Andrews, R. Askey, and R. Roy, Special Functions, vol. 71 of Encyclopedia of Mathematics and its Applications, Cambridge University Press, Cambridge, UK, 1999.

[2] Q.-M. Luo and H. M. Srivastava, "Some generalizations of the Apostol-Bernoulli and Apostol-Euler polynomials," Journal of Mathematical Analysis and Applications, vol. 308, no. 1, pp. 290302, 2005.
[3] B. Kurt, "A further generalization of the Bernoulli polynomials and on the $2 D$-Bernoulli polynomials $B_{n}^{2}(x, y)$," Applied Mathematics, vol. 233, pp. 3005-3017, 2010.

[4] Q.-M. Luo, " $q$-extensions for the Apostol-Genocchi polynomials," General Mathematics, vol. 17, no. 2, pp. 113-125, 2009.

[5] Q.-M. Luo, "Some results for the $q$-Bernoulli and $q$-Euler polynomials," Journal of Mathematical Analysis and Applications, vol. 363, no. 1, pp. 7-18, 2010.

[6] Q.-M. Luo, " $q$-analogues of some results for the Apostol-Euler polynomials," Advanced Studies in Contemporary Mathematics, vol. 20, no. 1, pp. 103-113, 2010.

[7] Q.-M. Luo and H. M. Srivastava, "Some relationships between the Apostol-Bernoulli and Apostol-Euler polynomials," Computers \& Mathematics with Applications, vol. 51, no. 3-4, pp. 631642, 2006.

[8] Q.-M. Luo and H. M. Srivastava, "Some generalizations of the Apostol-Genocchi polynomials and the Stirling numbers of the second kind," Applied Mathematics and Computation, vol. 217, no. 12, pp. 5702-5728, 2011.

[9] Q.-M. Luo, "An explicit relationship between the generalized Apostol-Bernoulli and Apostol-Euler polynomials associated with $\lambda$-Stirling numbers of the second kind," Houston Journal of Mathematics, vol. 36, no. 4, pp. 1159-1171, 2010.

[10] R. Tremblay, S. Gaboury, and B.-J. Fugère, "A new class of generalized Apostol-Bernoulli polynomials and some analogues of the Srivastava-Pintér addition theorem," Applied Mathematics Letters of Rapid Publication, vol. 24, no. 11, pp. 1888-1893, 2011.

[11] H. Ozden, Y. Simsek, and H. M. Srivastava, "A unified presentation of the generating functions of the generalized Bernoulli, Euler and Genocchi polynomials," Computers \& Mathematics with Applications, vol. 60, no. 10, pp. 2779-2787, 2010.

[12] D.-Q. Lu and H. M. Srivastava, "Some series identities involving the generalized Apostol type and related polynomials," Computers \& Mathematics with Applications, vol. 62, no. 9, pp. 35913602, 2011.

[13] H. M. Srivastava and J. Choi, Series Associated with the Zeta and Related Functions, Kluwer Academic, Dodrecht, The Netherlands, 2001.

[14] P. Natalini and A. Bernardini, "A generalization of the Bernoulli polynomials," Journal of Applied Mathematics, vol. 2003, no. 3, pp. 155-163, 2003.

[15] W. Wang, C. Jia, and T. Wang, "Some results on the ApostolBernoulli and Apostol-Euler polynomials," Computers \& Mathematics with Applications, vol. 55, no. 6, pp. 1322-1332, 2008.

[16] J. Choi, D. S. Jang, and H. M. Srivastava, "A generalization of the Hurwitz-Lerch Zeta function," Integral Transforms and Special Functions, vol. 19, no. 1-2, pp. 65-79, 2008.

[17] M. Garg, K. Jain, and H. M. Srivastava, "Some relationships between the generalized Apostol-Bernoulli polynomials and Hurwitz-Lerch Zeta functions," Integral Transforms and Special Functions, vol. 17, no. 11, pp. 803-815, 2006.

[18] M. Ali Özarslan, "Unified Apostol-Bernoulli, Euler and Genocchi polynomials," Computers \& Mathematics with Applications, vol. 62, no. 6, pp. 2452-2462, 2011.

[19] R. Dere and Y. Simsek, "Applications of umbral algebra to some special polynomials," Advanced Studies in Contemporary Mathematics, vol. 22, no. 3, pp. 433-438, 2012.

[20] B. Kurt and Y. Simsek, "Frobenious-Euler type polynomials related to Hermite-Bernoulli polynomials, analysis and applied math," AIP Conference Proceedings, vol. 1389, pp. 385-388, 2011. 
[21] N. I. Mahmudov, "On a class of $q$-Bernoulli and $q$-Euler polynomials," Advances in Difference Equations, vol. 2013, article 108, p. 11, 2013.

[22] N. I. Mahmudov and M. E. Keleshteri, "On a class of generalized $q$-Bernoulli and $q$-Euler polynomials," Advances in Difference Equations, vol. 2013, article 115, p. 10, 2013.

[23] T. N. T. Goodman, H. Oruç, and G. M. Phillips, "Convexity and generalized Bernstein polynomials," Proceedings of the Edinburgh Mathematical Society II, vol. 42, no. 1, pp. 179-190, 1999.

[24] N. I. Mahmudov, "Difference equations of $q$-Appell polynomials," http://arxiv.org/abs/1403.0189. 


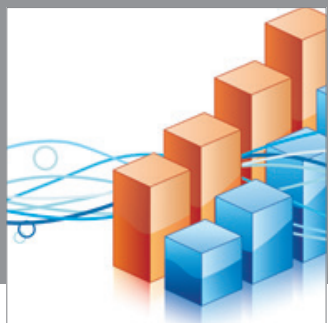

Advances in

Operations Research

mansans

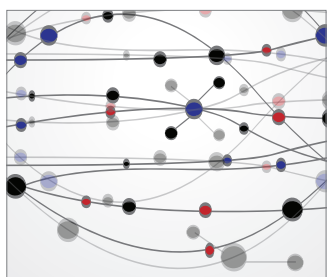

The Scientific World Journal
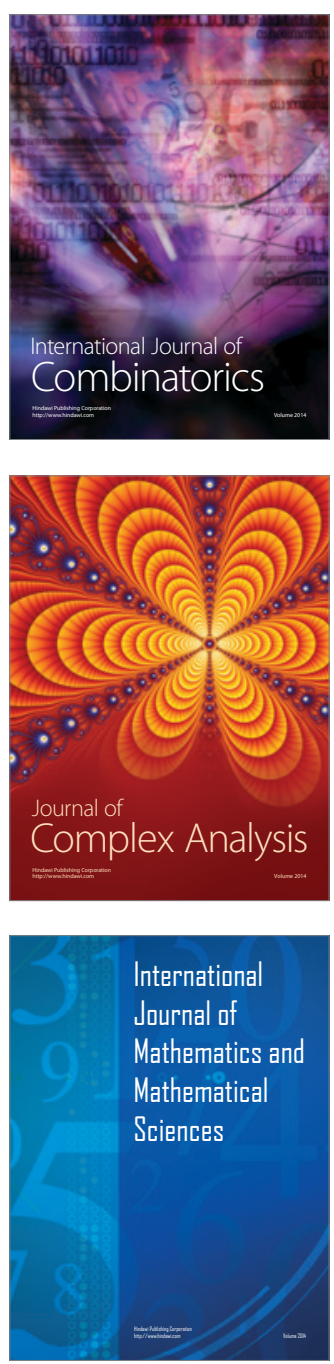
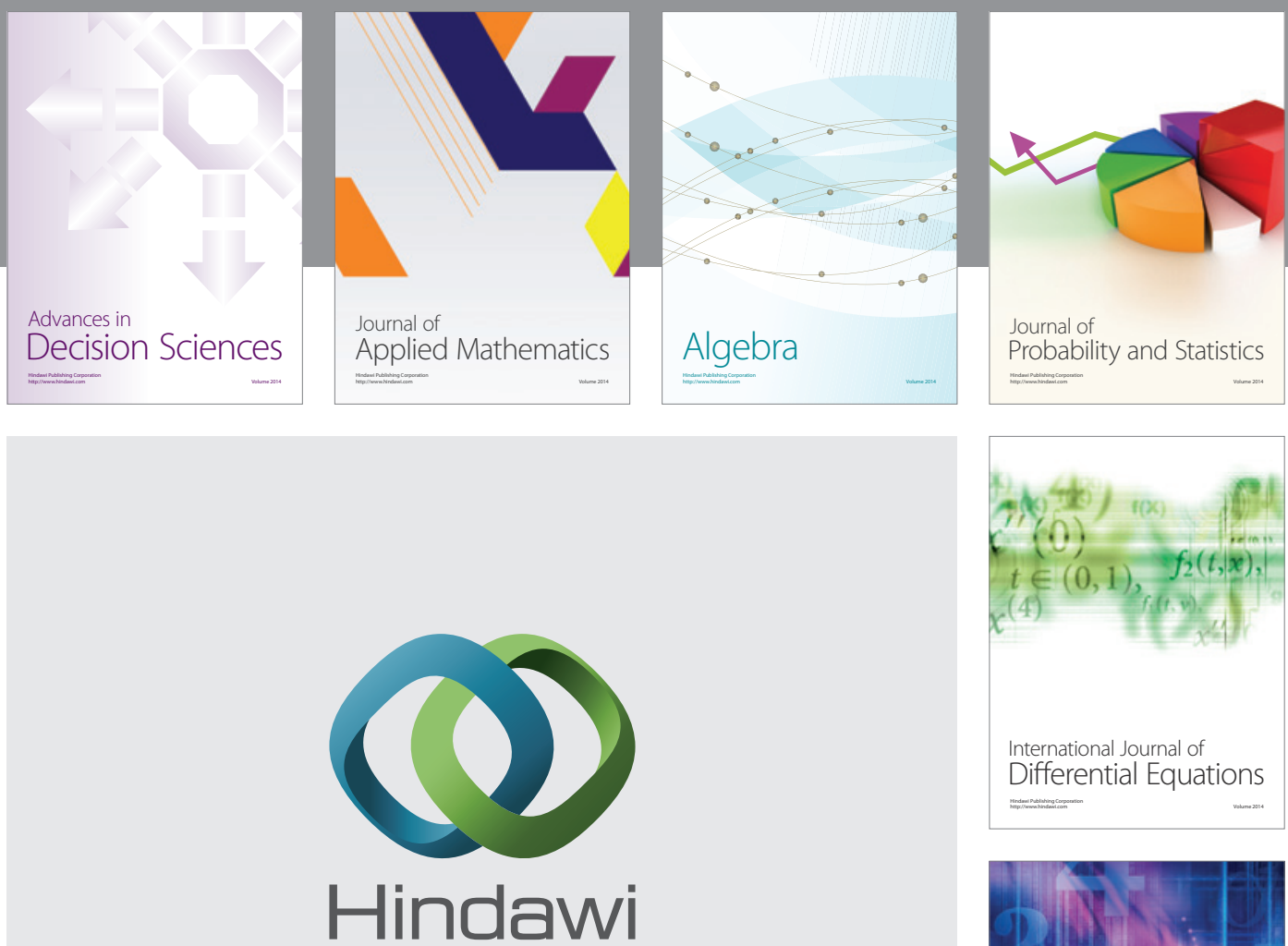

Submit your manuscripts at http://www.hindawi.com
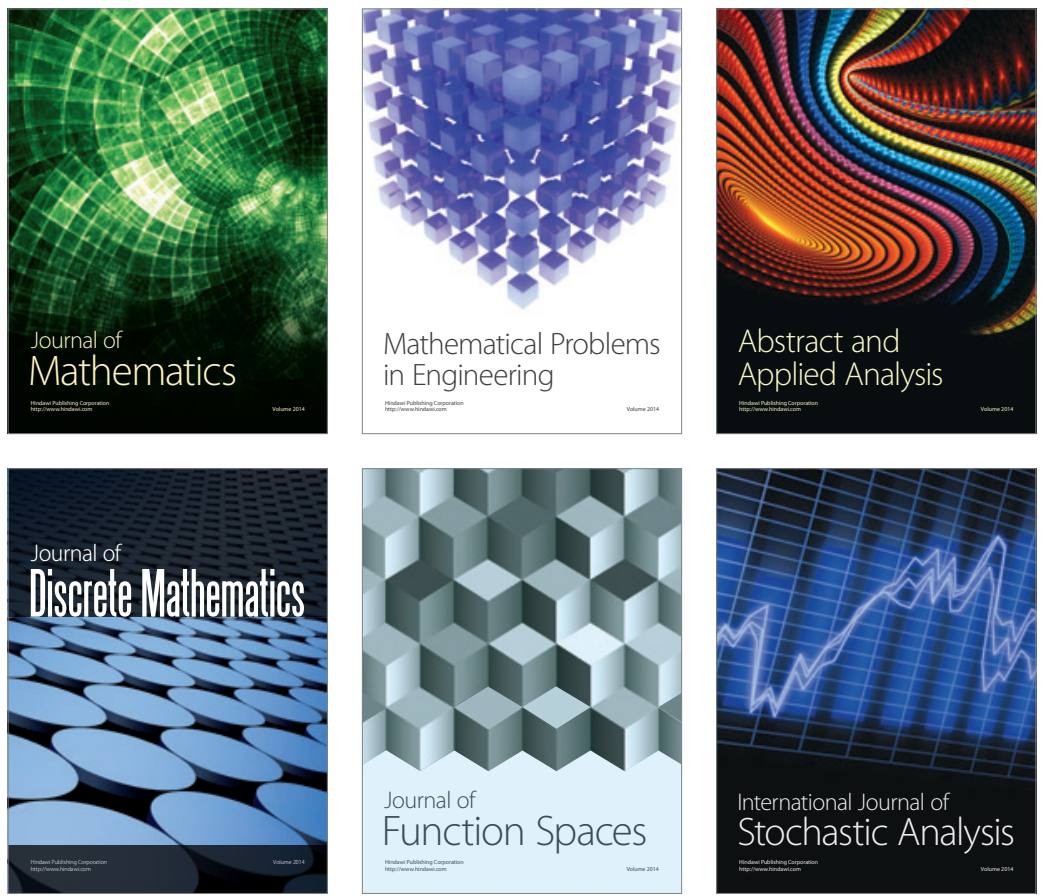

Journal of

Function Spaces

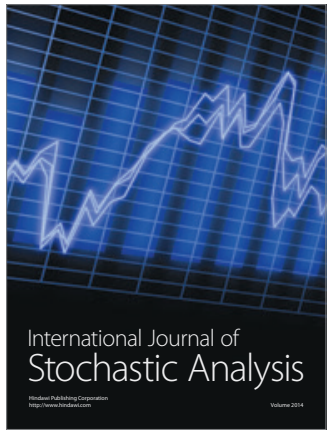


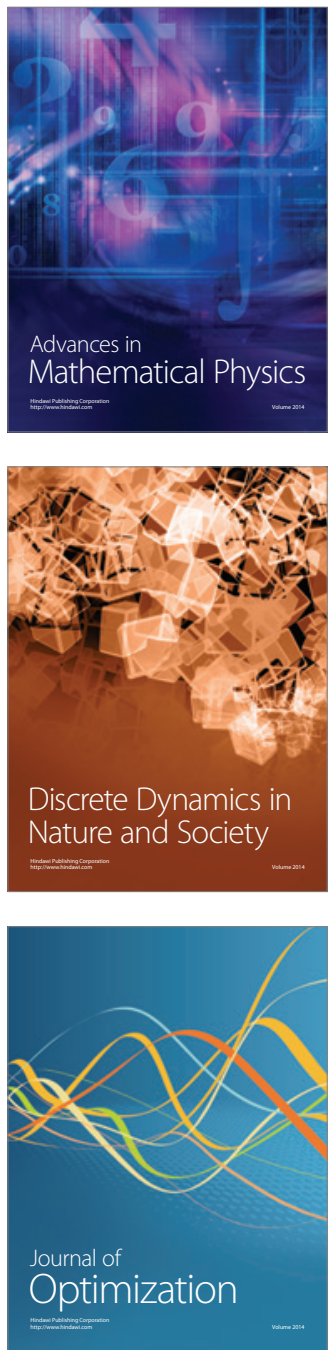